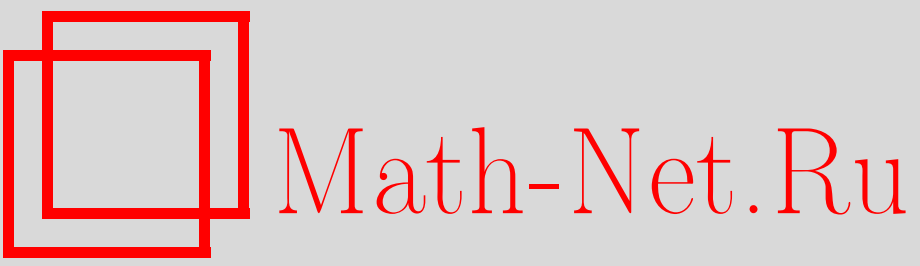

С. М. Клишевич, Гамильтонова формулировка массивных спинов 2 и 3 и замена переменных с одной производной, ТМФ, 1998, том 116, номер 2, 248-264

DOI: https://doi.org/10.4213/tmf901

Использование Общероссийского математического портала Math-Net.Ru подразумевает, что вы прочитали и согласны с пользовательским соглашением

http://www.mathnet.ru/rus/agreement

Параметры загрузки:

IP: 54.81 .137 .203

26 апреля 2023 г., 14:41:00 
ТЕОРЕТИЧЕСКАЯ

И МАТЕМАТИЧЕСКАЯ

ФИЗИКА

Том 116, № 2

август, 1998

(C) 1998 г.

С. М. Клишевич*

\section{ГАМИЛЬТОНОВА ФОРМУЛИРОВКА МАССИВНЫХ СПИНОВ 2 И 3 И ЗАМЕНА ПЕРЕМЕННЫХ С ОДНОЙ ПРОИЗВОДНОЙ}

Для массивных калибровочных полей со спинами 2 и 3 и неканоническими лагранжианами построены гамильтонианы и полные системы связей. Показано, что переопределение полей с использованием одной производной может являться преобразованием, изменяющим спектр теории.

\section{1. ВВЕДЕНИЕ}

При построении различных моделей в теории поля часто бывает удобно переопределять исходные поля, для того чтобы теория имела более простой вид. При таких заменах переменных число физических степеней свободы в теории должно сохраняться. При этом, конечно, имеется в виду, что не происходит изменения представлений группы Пуанкаре, т.е., к примеру, массивное векторное поле не переходит в три скалярных. Переопределения такого рода часто приходится делать в теориях, в которых физическая частица описьвается некоторым набором полей $[1,2]$. В работе [2] при изучении движения массивной частицы спина 2 в постоянном однородном электромагнитном поле с помошью переопределения поля второго ранга удалось получить результат, не зависящий от размерности пространства-времени.

Все замены переменных можно условно разбить на два типа. Первый тип - замена переменных без использования производных, что схематично можно записать в виде $\Phi_{A}^{\prime}=M_{A}^{B} \Phi_{B}+F_{A}^{B C} \Phi_{B} \Phi_{C}+\cdots$, где $M_{A}^{B}$ - невырожденная матрица. В классической теории поля такие преобразования не приводят к изменению числа физических степеней свободы, а в квантовой теории формулируется теорема [3-5], устанавливающая физическую эквивалентность обеих теорий. Второй тип - замена переменных с производными, когда $\Phi_{A}^{\prime}=M_{A}^{B} \Phi_{B}+H_{A}^{B} \partial \Phi_{B}+\cdots$. В классической теории в обшем случае такие преобразования приводят к изменению числа степеней свободы, соответственно в квантовом случае - к появлению духов, т.е. изменению спектра теории. В работе [6] такие преобразования были названы изменяющими спектр, и была предложена модифициро-

${ }^{*}$ Институт физики высоких энергий, Протвино, Московская обл., Россия. E-mail: klishevich@thl.ihep.su 
ванная формулировка теоремы эквивалентности, позволяюшая сохранить унитарность и спектр исходной теории.

В данной работе для свободных массивных полей спина 2 и 3 с помощью замен переменных как с производной, так и без нее мы пытаемся максимально упростить калибровочные преобразования. Используя гамильтонову формулировку, показываем, что для спина 3 замена переменных, приводяшая калибровочные преобразования к максимально простому виду, изменяет спектр. В то же время на примере простой механической системы показано, что преобразования такого типа не изменяют спектра (во всяком случае для неособенной теории).

Массивное поле со спином 2 возникает, например, в теориях типа Калуцы-Клейна [7], присутствует в спектре струны [8], а также при любой попытке рассмотреть массивную гравитацию [9]. Был проведен подробный гамильтонов анализ свободного спина 2 в пространстве Минковского [10]. Гамильтонова формулировка изучалась также в произвольном пространстве-времени Эйнштейна [11]. Однако во всех случаях рассматривалась калибровочно-неинвариантная формулировка для системы со спином 2, тогда как при размерной редукции и в случае струны описание должно быть калибровочно-инвариантно.

В разделе 2 данной работы мы рассматриваем свободное калибровочное массивное поле спина 2 в неканонической ${ }^{1)}$ форме, которая получается из канонической переопределением поля второго ранга. Мы строим канонический гамильтониан и полную систему связей ${ }^{2)}$ первого рода. Простой подсчет показывает, что при переходе к неканонической форме число физических степеней свободы не меняется, что можно рассматривать как иллюстрацию к теореме эквивалентности.

Массивное поле со спином 3 изучено в значительно меньшей степени. Состояние с таким спином присутствует в спектре струны. Его также можно рассматривать как естественный объект для описания аффинной теории гравитации. В разделе 3 рассматривается гамильтонова формулировка свободного массивного поля спина 3 в калибровочно-инвариантной форме для неканонического лагранжиана. Данное построение может оказаться полезным при гамильтоновом анализе теории взаимодействующего спина 3 , если такая будет построена.

В четвертом разделе на примере простой механической системы показано, что использование одной производной в замене переменных необязательно приводит к изменению спектра теории

В разделе 5 рассматривается гамильтонова формулировка теории, полученная из канонической формы теории для спина 3 переопределением полей с использованием производной. Построив полную систему связей, мы показываем, что в данном случае число полевых степеней свободы увеличивается на единицу. Это означает, что подобное преобразование изменяет спектр и при квантовании такой теории необходимо использовать модифицированную теорему эквивалентности [6].

\footnotetext{
1) Мы называем лагранжиан массивного поля спина $s$ каноническим, если в безмассовом пределе он распадается на сумму лагранжианов безмассовых полей спинов $s, s-1, \ldots, 0[2]$.

${ }^{2)}$ При описании систем со связями используется стандартная процедура Дирака $[12,13]$.
} 


\section{2. СВОБОДНОЕ МАССИВНОЕ ПОЛЕ СО СПИНОМ 2}

В этом разделе дана гамильтонова формулировка для калибровочного массивного поля со спином 2 с неканоническим лагранжианом.

Везде далее рассматривается плоское пространство Минковского $\mathbf{M}^{4}$ с сигнатурой метрики $(1,-1,-1,-1)$. Латинские индексы принимают значения $0,1,2,3$, греческие $1,2,3$. Мы также не будем делать различия между верхними и нижними индексами.

Свободное массивное поле со спином 2 можно описать калибровочно-инвариантным лагранжианом вида [2]

$$
\begin{aligned}
\mathcal{L}_{0}= & \partial_{m} \bar{h}_{k l} \partial_{m} h_{k l}-2 \partial_{k} h_{k l} \partial_{m} \bar{h}_{l m}+\left(\partial_{k} h_{k l} \partial_{l} \bar{h}+\text { h. c. }\right)-\partial_{k} \bar{h} \partial_{k} h+ \\
& +2\left(\partial_{k} \bar{h}_{k l} \partial_{l} \varphi-\partial_{l} \bar{h} \partial_{l} \varphi+\text { h.c. }\right)-2\left(\partial_{l} \bar{b}_{k} \partial_{l} b_{k}-\partial_{l} b_{k} \partial_{k} \bar{b}_{l}\right)+ \\
& +2 m\left(\partial_{l} \bar{b}_{k} h_{k l}-\partial_{k} \bar{b}_{k} h+\text { h.c. }\right)-m^{2}\left(\bar{h}_{k l} h_{k l}-\bar{h} h\right)
\end{aligned}
$$

где $h_{k l}$ - симметричный тензор, а $h=g^{k l} h_{k l}$.

Калибровочные преобразования для данного лагранжиана имеют следуюший вид:

$$
\begin{aligned}
\delta h_{k l} & =2 \partial_{(k} \xi_{l)}, \\
\delta b_{k} & =\partial_{k} \eta+m \xi_{k}, \\
\delta \varphi & =m \eta .
\end{aligned}
$$

Лагранжиан (1) выбран в неканоническом виде (с недиагональной кинетической частью), для того чтобы в преобразованиях (2) для поля $h_{k l}$ отсутствовало слагаемое голдстоуновского типа, пропорциональное массе. В канонической форме с данной нормировкой полей преобразования для $h$ имеют вид $\delta h_{k l}=2 \partial_{(k} \xi_{l)}+m g_{k l} \eta$, где $g_{k l}$ - метрический тензор. Следовательно, для перехода к лагранжиану (1) и преобразованиям (2) необходимо сделать следуюшее переопределение: $h^{\prime}{ }_{k l} \rightarrow h_{k l}-g_{k l} \varphi$.

Для удобства примем далее $m=1$.

Переходя от лагранжиана (1) к гамильтонову формализму, при вычислении импульсов получаем пять связей:

$$
\begin{aligned}
& \stackrel{(1)}{C}_{\alpha}^{h}=p_{\alpha 0}^{h}-\partial_{\alpha} h_{\beta \beta}+2 \partial_{\beta} h_{\alpha \beta}+2 \partial_{\alpha} \varphi+\partial_{\alpha} h_{00}, \\
& \stackrel{(1)}{C} h=p_{00}^{h}-\partial_{\alpha} h_{\alpha 0}, \\
& \stackrel{(1)}{C}^{b}=p_{0}^{b}-2 h_{\alpha \alpha} .
\end{aligned}
$$

Определим одновременные скобки Пуассона равенствами

$$
\begin{aligned}
\left\{h^{\alpha \beta}(x), p_{\mu \nu}^{h}(y)\right\} & =\delta_{(\mu \nu)}^{\alpha \beta}(x-y), \\
\left\{h^{\alpha 0}(x), p_{\beta 0}^{h}(y)\right\} & =\delta_{\beta}^{\alpha}(x-y), \\
\left\{h^{00}(x), p_{00}^{h}(y)\right\} & =\delta(x-y), \\
\left\{b^{\alpha}(x), p_{\beta}^{b}(y)\right\} & =\delta_{\beta}^{\alpha}(x-y), \\
\left\{b^{0}(x), p_{0}^{b}(y)\right\} & =\delta(x-y), \\
\left\{\varphi(x), p^{\varphi}(y)\right\} & =\delta(x-y),
\end{aligned}
$$


где принято обозначение $\delta_{\mu \nu \ldots}^{\alpha \beta \ldots}(x-y) \equiv \delta_{\mu}^{\alpha} \delta_{\nu \ldots}^{\beta \ldots} \delta(x-y)$.

Скобки Пуассона связей (3) между собой равны нулю. Гамильтониан, получающийся из (l), имеет следующий вид:

$$
\begin{aligned}
\mathcal{H}_{0}= & \bar{p}_{\alpha \beta}^{h} p_{\alpha \beta}^{h}-\frac{1}{3} \bar{p}_{\beta \beta}^{h} p_{\alpha \alpha}^{h}+\frac{1}{6} \bar{p}_{\alpha \alpha}^{h} p^{\varphi}+\frac{1}{6} p_{\alpha \alpha}^{h} \bar{p}^{\varphi}+\frac{1}{2} \bar{p}_{\alpha}^{b} p_{\alpha}^{b}+\frac{1}{6} \bar{p}^{\varphi} p^{\varphi}+ \\
& +\frac{1}{3} \partial_{\alpha} h_{\alpha 0} \bar{p}_{\beta \beta}^{h}+\frac{1}{3} \partial_{\alpha} \bar{h}_{\alpha 0} p_{\beta \beta}^{h}+\partial_{\alpha} \bar{b}_{0} p_{\alpha}^{b}+\partial_{\alpha} b_{0} \bar{p}_{\alpha}^{b}-\frac{1}{6} \partial_{\alpha} h_{\alpha 0} \bar{p}^{\varphi}- \\
& -\frac{1}{6} \partial_{\alpha} \bar{h}_{\alpha 0} p^{\varphi}+\bar{h}_{\alpha 0} p_{\alpha}^{b}+h_{\alpha 0} \bar{p}_{\alpha}^{b}-\partial_{\alpha} h_{00} \partial_{\beta} \bar{h}_{\alpha \beta}-\partial_{\beta} \bar{h}_{00} \partial_{\alpha} h_{\alpha \beta}+ \\
& +\partial_{\beta} h_{\alpha \alpha} \partial_{\beta} \bar{h}_{00}+\partial_{\alpha} h_{00} \partial_{\alpha} \bar{h}_{\beta \beta}+\frac{2}{3} \partial_{\alpha} h_{\alpha 0} \partial_{\beta} \bar{h}_{\beta 0}-2 \partial_{\beta} h_{\alpha 0} \partial_{\beta} \bar{h}_{\alpha 0}+ \\
& +\partial_{\gamma} h_{\alpha \beta} \partial_{\gamma} \bar{h}_{\alpha \beta}+\partial_{\beta} h_{\alpha \alpha} \partial_{\gamma} \bar{h}_{\beta \gamma}+\partial_{\beta} \bar{h}_{\gamma \gamma} \partial_{\alpha} h_{\alpha \beta}-\partial_{\beta} h_{\alpha \alpha} \partial_{\beta} \bar{h}_{\gamma \gamma}- \\
& -2 \partial_{\gamma} \bar{h}_{\beta \gamma} \partial_{\alpha} h_{\alpha \beta}-2 \partial_{\alpha} \varphi \partial_{\alpha} \bar{h}_{00}-2 \partial_{\alpha} \bar{\varphi} \partial_{\alpha} h_{00}+2 \partial_{\beta} h_{\alpha \alpha} \partial_{\beta} \bar{\varphi}+ \\
& +2 \partial_{\beta} \bar{h}_{\alpha \alpha} \partial_{\beta} \varphi-2 \partial_{\beta} \bar{\varphi} \partial_{\alpha} h_{\alpha \beta}-2 \partial_{\alpha} \bar{h}_{\alpha \beta} \partial_{\beta} \varphi+2 \partial_{\beta} b_{\alpha} \partial_{\beta} \bar{b}_{\alpha}- \\
& -2 \partial_{\beta} b_{\alpha} \partial_{\alpha} \bar{b}_{\beta}-2 \partial_{\alpha} b_{\alpha} \bar{h}_{00}-2 \partial_{\alpha} \bar{b}_{\alpha} h_{00}-2 \partial_{\beta} b_{\alpha} \bar{h}_{\alpha \beta}-2 \partial_{\beta} \bar{b}_{\alpha} h_{\alpha \beta}+ \\
& +2 \partial_{\alpha} b_{\alpha} \bar{h}_{\beta \beta}+2 \partial_{\alpha} \bar{b}_{\alpha} h_{\beta \beta}+4 \partial_{\alpha} \bar{b}_{0} h_{\alpha 0}+4 \partial_{\alpha} b_{0} \bar{h}_{\alpha 0}+\bar{h}_{\alpha \beta} h_{\alpha \beta}+ \\
& +\bar{h}_{00} h_{\alpha \alpha}+\bar{h}_{\alpha \alpha} h_{00}-\bar{h}_{\beta \beta} h_{\alpha \alpha},
\end{aligned}
$$

где $\gamma_{\alpha \beta}=-g_{\alpha \beta}, A_{\alpha \alpha \ldots} \equiv \gamma^{\alpha \beta} A_{\alpha \beta \ldots}$.

Для того чтобы уравнения Гамильтона были эквивалентны лагранжевым уравнениям, которые следуют из (1), необходимо к гамильтониану (5) добавить первичные связи с произвольными лагранжевыми множителями. Однако, т.к. связи "коммутируют" меж ду собой, то при вычислении вторичных связей они никакого вклада не дают.

Вычисляя эволюцию первичных связей (3), получаем пять вторичных связей:

$$
\begin{aligned}
& \stackrel{(2)}{C}_{\alpha}^{h}=2 \partial_{\beta} p_{\alpha \beta}^{h}-p_{\alpha}^{b}-2 \Delta h_{\alpha 0}-4 \partial_{\alpha} b_{0}, \\
& \stackrel{(2)}{C}^{h}=-\Delta h_{\alpha \alpha}+\partial_{\alpha \beta} h_{\alpha \beta}+2 \Delta \varphi-2 \partial_{\alpha} b_{\alpha}+h_{\alpha \alpha}, \\
& \stackrel{(2)}{C} b=\partial_{\alpha} p_{\alpha}^{b}-p^{\varphi}+2 \partial_{\alpha} h_{\alpha 0} .
\end{aligned}
$$

Скобки Пуассона вторичных связей между собой и с первичными связями равны нулю. Скобки между вторичными связями (6) и гамильтонианом (5) либо равны нулю, либо являются линейными комбинациями вторичных связей, т.е. на следующем этапе никаких новых связей не возникает. Следовательно, связи (3) и (6) образуют полную систему связей для данной теории. При этом все связи являются связями первого рода.

Нетрудно подсчитать, что число степеней свободы в данном случае равно пяти, что находится в согласии с формулой $2 s+1$ для массивной частицы произвольного спина $s$.

Для полного гамильтонова анализа необходимо наложить калибровки, вычислить скобки Дирака и перейти к редуцированному фазовому пространству, что выходит за рамки данной работы. 


\section{3. СВОБОДНОЕ МАССИВНОЕ ПОЛЕ СПИНА 3}

Так же как и в предыдущем разделе, мы будем описывать свободное массивное комплексное поле спина 3 калибровочно-инвариантным лагранжианом в "неканонической" форме:

$$
\begin{aligned}
\mathcal{L}_{0}= & -10 \partial_{n} \bar{\Phi}_{k l m} \partial_{n} \Phi_{k l m}+30 \partial_{k} \Phi_{k l m} \partial_{n} \bar{\Phi}_{l m n}-30\left(\partial_{k} \Phi_{k l m} \partial_{m} \bar{\Phi}_{l}+\text { h. c. }\right)+ \\
& +30 \partial_{l} \Phi_{k} \partial_{l} \bar{\Phi}_{k}+15 \partial_{l} \bar{\Phi}_{l} \partial_{k} \Phi_{k}-6\left(2 \partial_{k} \Phi_{k l m} \partial_{m} \bar{b}_{l}-2 \partial_{l} \Phi_{k} \partial_{l} \bar{b}_{k}-\right. \\
& \left.-\partial_{l} \bar{\Phi}_{l} \partial_{k} b_{k}+\text { h.c. }\right)+\frac{36}{5} \partial_{l} b_{k} \partial_{k} \bar{b}_{l}+30 \partial_{m} \bar{h}_{k l} \partial_{m} h_{k l}-60 \partial_{k} h_{k l} \partial_{m} \bar{h}_{l m}+ \\
& +30\left(\partial_{l} \bar{h} \partial_{k} h_{k l}+\text { h.c. }\right)-30 \partial_{k} h \partial_{k} \bar{h}+5\left(\partial_{l} \bar{\varphi} \partial_{k} h_{k l}-\partial_{k} h \partial_{k} \bar{\varphi}+\text { h.c. }\right)- \\
& -\frac{1}{4} \partial_{k} \bar{\varphi} \partial_{k} \varphi-15\left(2 \partial_{m} \bar{h}_{k l} \Phi_{k l m}-4 \partial_{k} \bar{h}_{k l} \Phi_{l}+\partial_{k} \bar{h} \Phi_{k}+\text { h.c. }\right)- \\
& -\frac{5}{2}\left(\partial_{k} \bar{\varphi} \Phi_{k}+\text { h. c. }\right)-18\left(\partial_{k} \bar{b}_{k} h+\text { h.c. }\right)+ \\
& +5\left(2 \bar{\Phi}_{k l m} \Phi_{k l m}-6 \bar{\Phi}_{k} \Phi_{k}+9 \bar{h} h\right),
\end{aligned}
$$

где $\Phi_{k l m}-$ симметричный тензор и $\Phi_{k}=g^{l m} \Phi_{k l m}$.

Преобразования для данного лагранжиана имеют следуюший вид:

$$
\begin{aligned}
\delta \Phi_{k l m} & =3 \partial_{(k} \omega_{l m)}-\frac{3}{5} g_{(k l} \partial_{m)} \eta \\
\delta h_{k l} & =2 \partial_{(k} \xi_{l)}+\omega_{k l}, \\
\delta b_{k} & =2 \partial_{k} \eta+5 \xi_{k}, \\
\delta \varphi & =12 \eta
\end{aligned}
$$

причем $g^{k l} \omega_{k l}=0$.

В канонической форме преобразования полей ранга 2 и 3 имели бы вид $\delta \Phi=\partial \omega+g \xi$ и $\delta h=\partial \xi+\omega+g \eta[1,2]$. Очевидно, что преобразования (8) имеют более простой вид, к тому же в преобразованиях для $\Phi$ отсутствует слагаемое голдстоуновского типа. Это значительно облегчает анализ теории при включении в нее взаимодействия. Переход от канонического вида к лагранжиану (7) и преобразованиям (8) достигается переопределением полей

$$
\Phi^{\prime} \rightarrow \Phi-g b, \quad h^{\prime} \rightarrow h-g \varphi .
$$

Приступим к гамильтоновой формулировке данной модели. Покажем также, что число степеней свободы при преобразованиях (8) не изменяется.

Вычисляя канонически-сопряженные импульсы, получаем 14 первичных связей:

$$
\begin{aligned}
& \stackrel{(1)}{C}^{b}=\frac{6}{5} p_{000}^{\Phi}-p_{0}^{b}-12 \partial_{\delta} \Phi_{\gamma \gamma \delta}+24 \partial_{\gamma} \Phi_{\gamma 00}+\frac{36}{5} \partial_{\gamma} b_{\gamma}, \\
& \stackrel{(1)}{C} h=-p_{00}^{h}-45 \Phi_{\gamma \gamma 0}+15 \Phi_{000}+30 \partial_{\gamma} h_{\gamma 0}+18 b_{0}, \\
& \stackrel{(1)}{C}{ }_{\alpha}^{h}=-p_{\alpha 0}^{h}+60 \Phi_{\alpha \gamma \gamma}-60 \partial_{\gamma} h_{\alpha \gamma}+30 \partial_{\alpha} h_{\gamma \gamma}-30 \partial_{\alpha} h_{00}-5 \partial_{\alpha} \varphi,
\end{aligned}
$$




$$
\begin{aligned}
\stackrel{(1)}{C}_{\alpha}^{\Phi}= & -p_{\alpha 00}^{\Phi}+30 \partial_{\gamma} \Phi_{\alpha \gamma 0}-30 \partial_{\alpha} \Phi_{\gamma \gamma 0}+30 \partial_{\alpha} \Phi_{000}+12 \partial_{\alpha} b_{0} \\
\stackrel{(1)}{C}_{\alpha \beta}^{\Phi}= & -p_{\alpha \beta 0}^{\Phi}-3 \gamma_{\alpha \beta} p_{000}^{\Phi}-30 \partial_{\gamma} \Phi_{\alpha \beta \gamma}+30 \partial_{(\alpha} \Phi_{\beta) \gamma \gamma}-30 \partial_{(\alpha} \Phi_{\beta) 00}+ \\
& +30 \gamma_{\alpha \beta} \partial_{\delta} \Phi_{\gamma \gamma \delta}-60 \gamma_{\alpha \beta} \partial_{\gamma} \Phi_{\gamma 00}-12 \partial_{(\alpha} b_{\beta)}-12 \gamma_{\alpha \beta} \partial_{\gamma} b_{\gamma}
\end{aligned}
$$

Для полей с рангом меньше 3 мы используем те же символы, что и в случае спина 2 , поэтому скобки Пуассона для этих переменных имеют вид (4). Для остальных переменных определим скобки следующим образом:

$$
\begin{aligned}
\left\{\Phi^{\alpha \beta \gamma}(x), p_{\lambda \mu \nu}^{\Phi}(y)\right\} & =\delta_{(\lambda \mu \nu)}^{\alpha \beta \gamma}(x-y), \\
\left\{\Phi^{\alpha \beta 0}(x), p_{\mu \nu 0}^{\Phi}(y)\right\} & =\delta_{(\mu \nu)}^{\alpha \beta}(x-y) \\
\left\{\Phi^{\alpha 00}(x), p_{\beta 00}^{\Phi}(y)\right\} & =\delta_{\beta}^{\alpha}(x-y) \\
\left\{\Phi^{000}(x), p_{000}^{\Phi}(y)\right\} & =\delta(x-y) .
\end{aligned}
$$

Скобки Пуассона всех первичных связей между собой равны нулю.

Теперь вычислим канонический гамильтониан. Даже для свободного поля получается достаточно громоздкий результат, поэтому конкретное выражение для гамильтониана мы вынесли в приложение А.

Условие сохранения первичных связей во времени дает вторичные связи:

$$
\begin{aligned}
\stackrel{(2)}{C}^{b}= & -\partial_{\gamma} p_{\gamma}^{b}-0.1 p_{\gamma \gamma}^{h}-5.4 b_{0}+6 p^{\varphi}-24 \partial^{2} \Phi_{\gamma \gamma 0}+12 \partial_{\gamma \delta}^{2} \Phi_{\gamma \delta 0}+ \\
& +12 \partial^{2} \Phi_{000}+7.2 \partial^{2} b_{0}-33 \partial_{\gamma} h_{\gamma 0}+13.5 \Phi_{\gamma \gamma 0}-4.5 \Phi_{000} \\
\stackrel{(2)}{C}^{h}= & 3 p_{000}^{\Phi}-30 \partial^{2} h_{\gamma \gamma}+30 \partial_{\gamma \delta}^{2} h_{\gamma \delta}+5 \partial^{2} \varphi-60 \partial_{\delta} \Phi_{\gamma \gamma \delta}+90 \partial_{\gamma} \Phi_{\gamma 00}+ \\
& +45 h_{\gamma \gamma}-45 h_{00}, \\
\stackrel{(2)}{C}_{\alpha}^{h}= & -2 \partial_{\gamma} p_{\alpha \gamma}^{h}+5 p_{\alpha}^{b}+60 \partial^{2} h_{\alpha 0}-60 \partial_{\gamma} \Phi_{\alpha \gamma 0}+30 \partial_{\alpha} \Phi_{\gamma \gamma 0}- \\
& -30 \partial_{\alpha} \Phi_{000}, \\
\stackrel{(2)}{C}_{\alpha}^{\Phi}= & 3 \partial_{\alpha} p_{000}^{\Phi}-30 \partial^{2} \Phi_{\alpha \gamma \gamma}+30 \partial_{\gamma \delta}^{2} \Phi_{\alpha \gamma \delta}-60 \partial_{\alpha \delta}^{2} \Phi_{\gamma \gamma \delta}+90 \partial_{\alpha \gamma}^{2} \Phi_{\gamma 00}+ \\
& +12 \partial^{2} b_{\alpha}+24 \partial_{\alpha \gamma}^{2} b_{\gamma}+30 \Phi_{\alpha \gamma \gamma}-60 \partial_{\gamma} h_{\alpha \gamma}+15 \partial_{\alpha} h_{\gamma \gamma}- \\
& -45 \partial_{\alpha} h_{00}-\frac{5}{2} \partial_{\alpha} \varphi, \\
\stackrel{(2)}{C}_{\alpha \beta}{ }_{\alpha \beta} & -3 \partial_{\gamma} p_{\alpha \beta \gamma}^{\Phi}+p_{\alpha \beta}^{h}+30 \partial^{2} \Phi_{\alpha \beta 0}-30 \partial_{\alpha \beta}^{2} \Phi_{000}+30 \partial_{\alpha \beta}^{2} \Phi_{\gamma \gamma 0}- \\
& -30 \gamma_{\alpha \beta} \partial_{\gamma \delta}^{2} \Phi_{\gamma 0}-30 \gamma_{\alpha \beta} \partial^{2} \Phi_{000}+60 \gamma_{\alpha \beta} \partial^{2} \Phi_{\gamma \gamma 0}-12 \partial_{\alpha \beta}^{2} b_{0}- \\
& -24 \gamma_{\alpha \beta} \partial^{2} b_{0}+60 \partial_{(\alpha} h_{\beta) 0}+90 \gamma_{\alpha \beta} \partial_{\gamma} h_{\gamma 0}-45 \gamma_{\alpha \beta} \Phi_{\gamma \gamma 0}+ \\
& +15 \gamma_{\alpha \beta} \Phi_{000}+18 \gamma_{\alpha \beta} b_{0} .
\end{aligned}
$$

Вторичные связи между собой и с первичными связями имеют нулевые скобки.

На третьем этапе никаких новых связей не возникает. Это означает, что связи (10) и (12) образуют полную систему, причем только первого рода. 
Теперь легко подсчитать число независимых полевых степеней свободы. Число всех полевых компонент равно 35, а число связей - 28. Следовательно, число независимых степеней свободы равно 7. Таким образом, при переходе к неканонической формулировке (7) с помощью замены переменных (9) число степеней свободы не изменилось.

\section{4. ЗАМЕНА ПЕРЕМЕННЫХ С ОДНОЙ ПРОИЗВОДНОЙ: ПРИМЕР МЕХАНИЧЕСКОЙ СИСТЕМЫ}

Рассмотрим на примере механической системы, описываемой координатами $\varphi^{\prime a}, a=$ $1, \ldots, N$, с произвольным лагранжианом $L\left(\varphi^{\prime}, \dot{\varphi}^{\prime}\right)$, преобразования переменных с одной производной по времени вида

$$
\varphi^{\prime a}=\varphi^{a}+R_{b}^{a} \dot{\varphi}^{b}
$$

где $R_{b}^{a}$ - произвольная постоянная матрица.

Если $R_{b}^{a}$ не пропорциональна матрице собственных нулевых векторов гессиана ${ }^{3)}$, то в лагранжиане появятся члены с высшими производными.

Перейдем к гамильтоновой форме, используя метод Остроградского [13-15]. Для этого введем следующие канонические переменные:

$$
\begin{aligned}
q_{1}^{a} & =\varphi^{a}, \quad q_{2}^{a}=\dot{\varphi}^{a}, \\
p_{1 a} & =\left(\frac{\partial}{\partial \dot{\varphi}^{a}}-\frac{d}{d t} \frac{\partial}{\partial \ddot{\varphi}^{a}}\right) L\left(\varphi^{\prime}(\varphi, \dot{\varphi}), \dot{\varphi}^{\prime}(\dot{\varphi}, \ddot{\varphi})\right)= \\
& =\frac{\partial L}{\partial \varphi^{\prime b}} \frac{\partial \varphi^{\prime b}}{\partial \dot{\varphi}^{a}}+\frac{\partial L}{\partial \dot{\varphi}^{\prime b}} \frac{\partial \dot{\varphi}^{\prime b}}{\partial \dot{\varphi}^{\prime a}}-\frac{d}{d t}\left(\frac{\partial L}{\partial \dot{\varphi}^{\prime b}} \frac{\partial \dot{\varphi}^{\prime b}}{\partial \ddot{\varphi}^{a}}\right)= \\
& =\frac{\partial L}{\partial \dot{\varphi}^{a}}+\left.R_{a}^{b} \frac{\delta L}{\delta \varphi^{\prime b}}\right|_{\varphi^{\prime}=\varphi+R \dot{\varphi}}, \\
p_{2 a} & =\frac{\partial}{\partial \ddot{\varphi}^{a}} L\left(\varphi^{\prime}(\varphi, \dot{\varphi}), \dot{\varphi}^{\prime}(\dot{\varphi}, \ddot{\varphi})\right)=\frac{\partial L}{\partial \dot{\varphi}^{\prime b}} \frac{\partial \dot{\varphi}^{\prime b}}{\partial \ddot{\varphi}^{a}}=R_{a}^{b} \frac{\partial L}{\partial \dot{\varphi}^{\prime a}} .
\end{aligned}
$$

Из (14) видно, что

$$
p_{2 a}=R_{a}^{b} p_{1 b}-R_{a}^{b} R_{b}^{c} \frac{\delta L}{\delta \varphi^{\prime c}}
$$

Если

$$
R_{a}^{b} R_{b}^{c} \frac{\delta L}{\delta \varphi^{\prime c}} \neq 0,
$$

то преобразования (13) изменяют спектр, т.к. в данном случае число степеней свободы увеличивается. В случае же, когда

$$
R_{a}^{b} R_{b}^{c} \frac{\delta L}{\delta \varphi^{\prime c}}=0
$$

появляются первичные связи

$$
\stackrel{(1)}{\Phi}_{a}=p_{2 a}-R_{a}^{b} p_{1 b}
$$

\footnotetext{
3) В случае вырожденной теории.
} 
Для того чтобы выяснить, меняется ли в этом случае спектр, мы должны вычислить все связи. Однако построение всех связей в общем случае выходит за рамки данной работы. Ограничимся рассмотрением простейшей механической системы.

Пусть механическая система описывается лагранжианом

$$
L=\frac{1}{2} \dot{q}_{1}^{2}+a \dot{q}_{1} \dot{q}_{2}+\frac{1}{2} \dot{q}_{1}^{2}-\frac{1}{2} m_{1}^{2} q_{1}^{2}-\frac{1}{2} m_{2}^{2} q_{1}^{2}
$$

где $a$ - произвольный параметр. Потребовав отсутствия "духов", получаем ограничение $1-a^{2} \geqslant 0$. Очевидно, что при $a^{2}=1$ имеем вырожденную систему.

Рассмотрим преобразование переменных вида (13) с нильпотентной матрицей $R_{a}^{b}$ :

$$
\left(\begin{array}{c}
q_{1} \\
q_{2}
\end{array}\right) \rightarrow\left(\begin{array}{c}
q_{1} \\
q_{2}
\end{array}\right)+\left(\begin{array}{cc}
0 & r \\
0 & 0
\end{array}\right)\left(\begin{array}{l}
\dot{q}_{1} \\
\dot{q}_{2}
\end{array}\right) .
$$

При этом условие (15) выполняется автоматически. После этой замены в лагранжиане появляются члены со старшими производными. Для работы с лагранжианами, имеющими старшие производные, будем в дальнейшем использовать способ, эквивалентный методу Остроградского, но, как нам кажется, более удобный в практических вычислениях, - понижение старших производных с помошью введения вспомогательных переменных.

После преобразования (17) и введения вспомогательной переменной $q_{3}=\dot{q}_{2}$ и лагранжева множителя $q_{4}$ получим лагранжиан

$$
\begin{aligned}
L= & \frac{1}{2} \dot{q}_{1}^{2}+r \dot{q}_{1} \dot{q}_{3}+\frac{1}{2} r^{2} \dot{q}_{3}^{2}+a \dot{q}_{1} q_{3}+a r \dot{q}_{3} q_{3}-\frac{1}{2} m_{1}^{2} q_{1}^{2}-\frac{1}{2} m_{2}^{2} q_{2}^{2}- \\
& -r m_{1}^{2} q_{1} q_{3}+\frac{1}{2}\left(1-r^{2} m_{1}^{2}\right) q_{3}^{2}+q_{4}\left(q_{3}-\dot{q}_{2}\right) .
\end{aligned}
$$

Поскольку в лагранжиане члены со старшими производными по времени уже не присутствуют, то для гамильтоновой формулировки можно использовать стандартную процедуру.

Канонические импульсы выражаются через скорости и координаты следующим образом:

$$
\begin{aligned}
& p_{1}=\dot{q}_{1}+r \dot{q}_{3}+a q_{3}, \\
& p_{2}=-q_{4}, \\
& p_{3}=r \dot{q}_{1}+r^{2} \dot{q}_{3}+a r q_{3}, \\
& p_{4}=0 .
\end{aligned}
$$

Канонический гамильтониан имеет вид

$$
\begin{aligned}
H_{\mathrm{c}}= & \sum_{i=1}^{4} p_{i} \dot{q}_{i}-L= \\
= & \frac{1}{2} p_{1}^{2}-a p_{1} q_{3}+\frac{1}{2}\left(m_{1}^{2} q_{1}^{2}+m_{2}^{2} q_{2}^{2}\right)+r m_{1}^{2} q_{1} q_{3}- \\
& \quad-\frac{1}{2}\left(1-a^{2}-r^{2} m_{1}^{2}\right) q_{3}^{2}-q_{3} q_{4} .
\end{aligned}
$$


Из соотношений (18) следует, что имеются три первичные связи:

$$
\stackrel{(1)}{C}{ }_{1}=p_{3}-r p_{1}, \quad \stackrel{(1)}{C}{ }_{2}=p_{2}+q_{4}, \quad \stackrel{(1)}{C}{ }_{3}=p_{4} .
$$

Соответственно полный гамильтониан имеет вид

$$
H_{\mathrm{tot}}=H_{\mathrm{c}}+\sum_{i=1}^{3} \lambda_{i} \stackrel{(1)}{C}_{i}
$$

Условие сохранения связей во времени фиксирует два лагранжевых множителя $\lambda_{2}=q_{3}$, $\lambda_{3}=m_{2}^{2} q_{2}$, а также дает вторичную связь

$$
\stackrel{(2)}{C}=\left\{\stackrel{(1)}{C}_{1}, H_{\text {tot }}\right\}=a p_{1}+\left(1-a^{2}\right) q_{3}+q_{4} .
$$

Условие сохранения вторичной связи во времени приводит к соотношению

$$
\left\{\stackrel{(2)}{C}, H_{\text {tot }}\right\}=\left(1-a^{2}\right) \lambda_{1}-a m_{1}^{2} q_{1}+m_{2}^{2} q_{2}-a r m_{1}^{2} q_{3} \approx 0 .
$$

Дальше ситуация зависит от того, вырождена механическая система или нет.

Сначала рассмотрим случай невырожденной системы, когда $1-a^{2}>0$. В этом случае условие (20) определяет лагранжев множитель $\lambda_{1}$ и больше связей в системе не возникает, причем все имеющиеся связи второго рода. Подсчитаем число степеней свободы: 8 импульсов и координат плюс 4 связи дают две степени свободы, т.е. столько же, сколько и было в теории с лагранжианом (16) до преобразования (17).

Редуцированный гамильтониан

$$
H_{\mathrm{red}}=\frac{1}{2} p_{1}^{2}+\frac{1}{2} \frac{\left(p_{2}-a p_{1}\right)^{2}}{1-a^{2}}+\frac{1}{2} m_{1}^{2}\left(q_{1}+r \frac{p_{2}-a p_{1}}{1-a^{2}}\right)^{2}+\frac{1}{2} m_{2}^{2} q_{2}^{2}
$$

положительно-определен, т. е. преобразование (17) не привело ни к изменению степеней свободы, ни к появлению “духов".

При наличии вырождения $\left(a^{2}=1\right)$ условие $(20)$ означает появление связи третьего этапа

$$
\stackrel{(3)}{C}=a m_{1}^{2}\left(q_{1}+r q_{3}\right)-m_{2}^{2} q_{2}
$$

Эта связь имеет нулевые скобки Пуассона с $\stackrel{(1)}{C}$, поэтому условие инволюции во времени для $\stackrel{(3)}{C}$ приводит к появлению связи четвертого этапа:

$$
\stackrel{(4)}{C}=\left\{\stackrel{(3)}{C}, H_{\text {tot }}\right\}=a m_{1}^{2} p_{1}-\left(m_{1}^{2}+m_{2}^{2}\right) q_{3} .
$$

Так как $\left\{\stackrel{(1)}{C}{ }_{1}, \stackrel{(4)}{C}\right\}=m_{1}^{2}+m_{2}^{2}$, то на следуюшем этапе новой связи не возникает, лишь определяется последний лагранжев множитель $\lambda_{1}=-\left(a m_{1}^{4} /\left(m_{1}^{2}+m_{2}^{2}\right)\right)\left(q_{1}+r q_{3}\right)$. Нетрудно проверить, что все имеюшиеся связи являются связями второго рода. 
Редуцированный гамильтониан в этом случае имеет вид

$$
H_{\text {red }}=\frac{1}{2} p_{1}^{2}+\frac{1}{2} m_{1}^{2}\left(\frac{m_{1}^{2}+m_{2}^{2}}{m_{2}^{2}}\right)\left(q_{1}+a r\left(\frac{m_{1}^{2}}{m_{1}^{2}+m_{2}^{2}}\right) p_{1}\right)^{2} .
$$

Этот гамильтониан также положительно-определен. Это означает, что и при наличии вырож дения преобразования (17) не привели ни к изменению степеней свободы, ни к появлению “духов", следовательно, при таком преобразовании можно пользоваться обычной теоремой эквивалентности.

Таким образом, мы показали, что преобразования с одной производной необязательно приводят к появлению лишних степеней свободы или “духов" . В следуюшем разделе мы покажем, что преобразования вида (13) с нильпотентной матрицей $R_{a}^{b}$ в случае массивного калибровочного поля со спином 3 могут привести к появлению лишних степеней свободы.

\section{5. ЗАМЕНА ПЕРЕМЕННЫХ С ОДНОЙ ПРОИЗВОДНОЙ: МАССИВНЫЙ СПИН 3}

В калибровочной теории массивных полей высоких спинов часто бывает удобным делать некоторые переопределения полей, для того чтобы привести калибровочные преобразования к более простому виду $[2,16]$. Хотелось бы также использовать и класс замен переменных с одной производной, который не меняет тип калибровочных преобразований ${ }^{4)}$. Как нетрудно убедиться, в случае массивных спинов 1 и 2, описываемых наборами полей $\left\{A_{k}, \phi\right\}$ и $\left\{h_{k l}, b_{k}, \varphi\right\}$, соответственно, общий вид замен переменных такого вида есть $A_{k} \rightarrow A_{k}+\partial_{k} \phi, h_{k l} \rightarrow h_{k l}+\partial_{(k} b_{l)}, b_{k} \rightarrow b_{k}+\partial_{k} \varphi$. Эти преобразования принадлежат к виду (13) с матрицей $R$, пропорциональной матрице собственных векторов гессиана, и поэтому не приводят к появлению в лагранжиане старших производных и соответственно не меняют спектра теории. Однако уже в случае массивного поля со спином 3 дело обстоит иначе. Перейдем к рассмотрению данного случая.

Нетрудно заметить, что преобразования (8) можно привести к более простому виду, сделав сдвиг поля третьего ранга

$$
\Phi^{\prime} \rightarrow \Phi+g \partial \varphi
$$

так, чтобы для $\Phi$ остались только преобразования вида $\partial \omega$. Кроме упрошения преобразований, мы получили бы еше одно преимушество. Поскольку после такого сдвига в преобразованиях полей отсутствует метрический тензор, то лагранжиан не зависит от размерности пространства-времени.

Сдвиг (21) принадлежит к преобразованиям типа (13) с нильпотентной матрицей. В предыдущем разделе мы выяснили, что такие преобразования не обязательно приводят к изменению спектра теории. Покажем, что переопределение полей вида (21) в данном случае приводит к увеличению числа степеней свободы на единицу.

\footnotetext{
${ }^{4)}$ То есть, например, замены, не приводящие к появлению в преобразованиях членов типа $\partial^{2} \xi$.
} 
После рассмотренного преобразования в лагранжиане появляются члены со старшими производными. Прежде чем перейти к гамильтоновой формулировке, понизим число производных в лагранжиане с помошью введения вспомогательного поля $v_{k}$. Далее будем действовать обычным образом.

После замены переменных и введения вспомогательного поля получим следующий лагранжиан:

$$
\begin{aligned}
\mathcal{L}_{0}= & \left(2 \partial_{m} \Phi_{k l m} \partial_{l} \bar{v}_{k}-2 \partial_{l} \Phi_{k} \partial_{l} \bar{v}_{k}+2 \partial_{l} \Phi_{k} \partial_{k} \bar{v}_{l}-3 \partial_{l} \Phi_{l} \partial_{m} \bar{v}_{m}+\text { h. c. }\right)- \\
& -10 \partial_{n} \Phi_{k l m} \partial_{n} \bar{\Phi}_{k l m}+30 \partial_{n} \Phi_{k l n} \partial_{m} \bar{\Phi}_{k l m}-30\left(\partial_{n} \Phi_{k m n} \partial_{m} \bar{\Phi}_{k}+\text { h.c. }\right)+ \\
& +30 \partial_{m} \Phi_{k} \partial_{m} \bar{\Phi}_{k}+15 \partial_{m} \Phi_{m} \partial_{k} \bar{\Phi}_{k}-6\left(2 \partial_{m} \Phi_{k l m} \partial_{l} \bar{b}_{k}-2 \partial_{m} b_{l} \partial_{m} \bar{\Phi}_{l}-\right. \\
& \left.-\partial_{m} b_{m} \partial_{l} \bar{\Phi}_{l}+\text { h.c. }\right)+30 \partial_{m} h_{k l} \partial_{m} \bar{h}_{k l}-60 \partial_{m} h_{k m} \partial_{l} \bar{h}_{k l}+ \\
& +30\left(\partial_{m} h_{l m} \partial_{l} \bar{h}+\text { h.c. }\right)-30 \partial_{l} h \partial_{l} \bar{h}-15\left(2 \partial_{m} h_{k l} \bar{\Phi}_{k l m}-\right. \\
& \left.-4 \partial_{m} h_{k m} \bar{\Phi}_{k}+\partial_{k} h \bar{\Phi}_{k}+\text { h.c. }\right)+\left(\bar{\lambda}_{k}\left(\partial_{k} \varphi-v_{k}\right)+\text { h. c. }\right)+ \\
& +10 \Phi_{k l m} \bar{\Phi}_{k l m}-30 \Phi_{k} \bar{\Phi}_{k} .
\end{aligned}
$$

Соответственно калибровочные преобразования (8) после сдвига (21) и введения вспомогательного поля примут вид

$$
\begin{aligned}
\delta \Phi_{k l m} & =3 \partial_{(k} \omega_{l m)}, \\
\delta h_{k l} & =2 \partial_{(k} \xi_{l)}+\omega_{k l} \\
\delta b_{k} & =2 \partial_{k} \eta+5 \xi_{k}, \\
\delta v_{k} & =12 \partial_{k} \eta \\
\delta \varphi & =12 \eta .
\end{aligned}
$$

Переходя к гамильтоновой формулировке теории с лагранжианом (22), получаем на первом этапе следующие связи:

$$
\begin{aligned}
& \stackrel{(1)}{C}^{\lambda}=p_{0}^{\lambda}, \\
& \stackrel{(1)}{C}_{\alpha}^{\lambda}=p_{\alpha}^{\lambda}, \\
& \stackrel{(1)}{C} \varphi=p^{\varphi}-\lambda_{0}, \\
& \stackrel{(1)}{C} v=-\frac{1}{6} p_{0}^{b}-p_{0}^{v}-2 \partial_{\delta} \Phi_{\gamma \gamma \delta}+2 \partial_{\gamma} \Phi_{\gamma 00}, \\
& \stackrel{(1)}{C}_{\alpha}^{v}=-\frac{1}{6} p_{\alpha}^{b}-p_{\alpha}^{v}+2 \partial_{\alpha} \Phi_{\gamma \gamma 0}-2 \partial_{\alpha} \Phi_{000}, \\
& \stackrel{(1)}{C} h=-p_{00}^{h}-45 \Phi_{\gamma \gamma 0}+15 \Phi_{000}+30 \partial_{\gamma} h_{\gamma 0}, \\
& \stackrel{(1)}{C}_{\alpha}^{h}=-p_{\alpha 0}^{h}+60 \Phi_{\alpha \gamma \gamma}-60 \partial_{\gamma} h_{\alpha \gamma}+30 \partial_{\alpha} h_{\gamma \gamma}-30 \partial_{\alpha} h_{00}, \\
& \stackrel{(1)}{C}_{\alpha}^{\Phi}=-p_{\alpha 00}^{\Phi}+30 \partial_{\gamma} \Phi_{\alpha \gamma 0}-30 \partial_{\alpha} \Phi_{\gamma \gamma 0},
\end{aligned}
$$




$$
\begin{aligned}
\stackrel{(1)}{C}_{\alpha \beta}^{\Phi}= & -p_{\alpha \beta 0}^{\Phi}-3 p_{000}^{\Phi} \gamma_{\alpha \beta}-30 \partial_{\gamma} \Phi_{\alpha \beta \gamma}-30 \partial_{(\alpha} \Phi_{\beta) 00}+30 \partial_{(\alpha} \Phi_{\beta) \gamma \gamma}+ \\
& +30 \partial_{\delta} \Phi_{\gamma \gamma \delta} \gamma_{\alpha \beta}-60 \partial_{\gamma} \Phi_{\gamma 00} \gamma_{\alpha \beta}-12 \partial_{(\alpha} b_{\beta)}-12 \partial_{\gamma} b_{\gamma} \gamma_{\alpha \beta}+ \\
& +2 \partial_{(\alpha} v_{\beta)}+6 \partial_{\gamma} v_{\gamma} \gamma_{\alpha \beta} .
\end{aligned}
$$

Так как в лагранжиане (22), в отличие от лагранжиана (7), появились дополнительные переменные - вспомогательное поле $v_{k}$ и лагранжев множитель $\lambda_{k}$, то скобки Пуассона (4) и (11) необходимо доопределить:

$$
\begin{aligned}
\left\{v_{0}(x), p_{0}^{v}(y)\right\} & =\delta(x-y), \\
\left\{v_{\alpha}(x), p_{\beta}^{v}(y)\right\} & =\delta_{\alpha \beta}(x-y), \\
\left\{\lambda_{0}(x), p_{0}^{\lambda}(y)\right\} & =\delta(x-y), \\
\left\{\lambda_{\alpha}(x), p_{\beta}^{\lambda}(y)\right\} & =\delta_{\alpha \beta}(x-y) .
\end{aligned}
$$

Среди первичных связей только две имеют ненулевые скобки:

$$
\left\{\stackrel{(1)}{C^{\lambda}}(x), \stackrel{(1)}{C}^{v}(y)\right\}=\delta(x-y),
$$

т.е., кроме связей первого рода, в теории появляются и связи второго рода.

Плотность канонического гамильтониана, которая получается в данном случае, приведена в приложении Б.

Из условия сохранения первичных связей во времени находим вторичные связи:

$$
\begin{aligned}
\stackrel{(1)}{\Lambda}_{0}^{\lambda}= & \partial_{\alpha} \lambda_{\alpha} \\
\stackrel{(1)}{\Lambda}^{\varphi}= & v_{0} \\
\stackrel{(2)}{C}_{\alpha}^{\lambda}= & -\partial_{\alpha} \varphi+v_{\alpha}, \\
\stackrel{(2)}{C}^{v}= & \lambda_{0}, \\
\stackrel{(2)}{C}_{\alpha}^{v}= & \lambda_{\alpha} \\
\stackrel{(2)}{C}^{h}= & \frac{5}{2} p_{0}^{b}-30 \partial^{2} h_{\gamma \gamma}+30 \partial_{\gamma \delta}^{2} h_{\gamma \delta}-30 \partial_{\delta} \Phi_{\gamma \gamma \delta}+30 \partial_{\gamma} \Phi_{\gamma 00}, \\
\stackrel{(2)}{C}_{\alpha}^{h}= & -2 \partial_{\gamma} p_{\alpha \gamma}^{h}+5 p_{\alpha}^{b}+60 \partial^{2} h_{\alpha 0}-60 \partial_{\gamma} \Phi_{\alpha \gamma 0}+30 \partial_{\alpha} \Phi_{\gamma \gamma 0}- \\
& -30 \partial_{\alpha} \Phi_{000}, \\
\stackrel{(2)}{C}_{\alpha}^{\Phi}= & +3 \partial_{\alpha} p_{000}^{\Phi}-30 \partial^{2} \Phi_{\alpha \gamma \gamma}+30 \partial_{\gamma \delta}^{2} \Phi_{\alpha \gamma \delta}-60 \partial_{\alpha \delta}^{2} \Phi_{\gamma \gamma \delta}+90 \partial_{\alpha \gamma}^{2} \Phi_{\gamma 00}+ \\
& +12 \partial^{2} b_{\alpha}+24 \partial_{\alpha \gamma}^{2} b_{\gamma}-2 \partial^{2} v_{\alpha}-10 \partial_{\alpha \gamma}^{2} v_{\gamma}+30 \Phi_{\alpha \gamma \gamma}- \\
& -60 \partial_{\gamma} h_{\alpha \gamma}+15 \partial_{\alpha} h_{\gamma \gamma}-45 \partial_{\alpha} h_{00}, \\
\stackrel{(2)}{C}_{\alpha \beta}^{\Phi}= & -3 \partial_{\gamma} p_{\alpha \beta \gamma}^{\Phi}+p_{\alpha \beta}^{h}+30 \partial^{2} \Phi_{\alpha \beta 0}-30 \partial_{\alpha \beta}^{2} \Phi_{000}+30 \partial_{\alpha \beta}^{2} \Phi_{\gamma \gamma 0}+
\end{aligned}
$$




$$
\begin{aligned}
& +60 \gamma_{\alpha \beta} \partial^{2} \Phi_{\gamma \gamma 0}-30 \gamma_{\alpha \beta} \partial^{2} \Phi_{000}-30 \gamma_{\alpha \beta} \partial_{\gamma \delta}^{2} \Phi_{\gamma \delta 0}-12 \partial_{\alpha \beta}^{2} b_{0}- \\
& -24 \gamma_{\alpha \beta} \partial^{2} b_{0}+6 \partial_{\alpha \beta}^{2} v_{0}+6 \gamma_{\alpha \beta} \partial^{2} v_{0}+60 \partial_{(\alpha} h_{\beta) 0}+ \\
& +90 \gamma_{\alpha \beta} \partial_{\gamma} h_{\gamma 0}+15 \gamma_{\alpha \beta} \Phi_{000}-45 \gamma_{\alpha \beta} \Phi_{\gamma \gamma 0}
\end{aligned}
$$

Первичные и вторичные связи, помимо (26), имеют следуюшие ненулевые скобки Пуассона:

$$
\begin{aligned}
& \left\{\stackrel{(1)}{C}^{\lambda}(x), \stackrel{(2)}{C}^{v}(y)\right\}=\delta(x-y), \\
& \left\{\stackrel{(1)}{C}^{\varphi}(x) \stackrel{(2)}{C}_{\alpha}^{\lambda}(y)\right\}=\partial_{\alpha}^{y} \delta(x-y), \\
& \left\{\stackrel{(1)}{C} v(x) \stackrel{(2)}{C}_{\beta}^{\lambda}(y)\right\}=\delta_{\alpha \beta}(x-y), \\
& \left\{\stackrel{(1)}{C}_{\alpha}^{\lambda}(x) \stackrel{(2)}{C}_{\beta}^{v}(y)\right\}=-\delta_{\alpha \beta}(x-y),
\end{aligned}
$$

где $\partial_{\alpha}^{y}=\partial / \partial y_{\alpha}$.

На третьем этапе новых связей не возникает, но происходит частичное определение лагранжевых множителей:

$$
\Lambda_{\alpha}^{v}=-\partial_{\alpha} v_{0}, \quad \Lambda_{\alpha}^{\lambda}=0
$$

Всего у нас имеется 15 “некоммутируюших" связей - первичные связи $\stackrel{(1)}{C} \varphi \stackrel{(1)}{C} \alpha, \stackrel{(1)}{C} \underset{\alpha}{\lambda}$, $\stackrel{(1)}{C} \underset{\alpha}{v}$ и вторичные связи $\stackrel{(2)}{C} \underset{\alpha}{\lambda}, \stackrel{(2)}{C} \varphi, \stackrel{(2)}{C} \underset{\alpha}{\varphi}$. Среди этих связей сушествует линейная комбинация, которая имеет нулевые скобки со всеми связями, т.е. является связью первого рода:

$$
\stackrel{(1)}{C}^{c}+\partial_{\alpha} \stackrel{(1)}{C}_{\alpha}^{v}+\stackrel{(2)}{C}^{v}
$$

Таким образом, среди пятнадшати “некоммутируюших" связей четырнадшать являются связями второго рода.

Вычислив алгебру связей, подсчитаем число степеней свободы. Число всех полевых степеней свободы в теории с лагранжианом (22) равно $20+10+4+1+4+4=43$. При этом имеются 22 первичные и 20 вторичных связей. Из них 14 связей второго и 28 первого рода. Следовательно, число степеней свободы в данной теории равно $43-$ $28-14 / 2=8$, а не 7, как должно быть в теории, описывающей свободную массивную частицу спина 3.

Таким образом, можно сделать вывод, что переопределения полей с использованием одной производной вида $\varphi_{a} \rightarrow \varphi_{a}+R_{a}^{b} \dot{\varphi}_{b}$ с нильпотентной матрицей $R_{a}^{b}$ в случае массивного поля со спином 3 могут являться преобразованиями, изменяющими спектр. Соответственно при квантовании такой теории необходимо использовать модифицированную теорему эквивалентности [6].

\section{ЗАКЛЮЧЕНИЕ}

В данной работе дана гамильтонова формулировка для свободных калибровочных массивных полей со спинами 2 и 3 и показано, что преобразования полей вида (13) с 
нильпотентной матрицей могут изменять спектр. Это означает, что при использовании замен переменных такого типа необходимо тщательно следить за тем, не изменяют ли они спектр, для того чтобы правильно проквантовать теорию.

Автор благодарен Ю. М. Зиновьеву и А. В. Разумову за обсуждение и помощь в работе, а также рецензенту за указание на существование модифицированной теоремы эквивалентности и конструктивные замечания.

ПРИЛОЖКНИЕ А

Каноническая плотность гамильтониана в случае лагранжиана (7) имеет вид

$$
\begin{aligned}
& \mathcal{H}=\frac{1}{5} \bar{p}_{000}^{\Phi} p_{000}^{\Phi}+\frac{1}{10} \bar{p}_{\alpha \beta \gamma}^{\Phi} p_{\alpha \beta \gamma}^{\Phi}-\frac{3}{50} \bar{p}_{\beta \gamma \gamma}^{\Phi} p_{\alpha \alpha \beta}^{\Phi}+\frac{1}{20} \bar{p}_{\alpha \alpha \beta}^{\Phi} p_{\beta}^{b}+\frac{1}{20} \bar{p}_{\beta}^{b} p_{\alpha \alpha \beta}^{\Phi}+ \\
& +\frac{1}{30} \bar{p}_{\alpha \beta}^{h} p_{\alpha \beta}^{h}-\frac{7}{720} \bar{p}_{\beta \beta}^{h} p_{\alpha \alpha}^{h}+\frac{1}{12} \bar{p}_{\alpha \alpha}^{h} p^{\varphi}+\frac{1}{12} p_{\alpha \alpha}^{h} \bar{p}^{\varphi}+\frac{1}{6} \bar{p}_{\alpha}^{b} p_{\alpha}^{b}+\bar{p}^{\varphi} p^{\varphi}- \\
& -3 \partial_{\beta} \Phi_{\alpha \alpha \beta} \bar{p}_{000}^{\Phi}-3 \partial_{\beta} \bar{\Phi}_{\alpha \alpha \beta} p_{000}^{\Phi}+3 \partial_{\alpha} \Phi_{\alpha 00} \bar{p}_{000}^{\Phi}+3 \partial_{\alpha} \bar{\Phi}_{\alpha 00} p_{000}^{\Phi}+ \\
& +\frac{3}{5} \partial_{\alpha} \Phi_{\alpha \beta 0} \bar{p}_{\beta \gamma \gamma}^{\Phi}+\frac{3}{5} \partial_{\alpha} \bar{\Phi}_{\alpha \beta 0} p_{\beta \gamma \gamma}^{\Phi}+\frac{6}{5} \partial_{\alpha} b_{\alpha} \bar{p}_{000}^{\Phi}+\frac{6}{5} \partial_{\alpha} \bar{b}_{\alpha} p_{000}^{\Phi}+ \\
& +\frac{9}{25} \partial_{\alpha} b_{0} \bar{p}_{\alpha \beta \beta}^{\Phi}+\frac{9}{25} \partial_{\alpha} \bar{b}_{0} p_{\alpha \beta \beta}^{\Phi}+\frac{7}{24} \partial_{\alpha} h_{\alpha 0} \bar{p}_{\beta \beta}^{h}+\frac{7}{24} \partial_{\alpha} \bar{h}_{\alpha 0} p_{\beta \beta}^{h}- \\
& -\frac{1}{2} \partial_{\alpha} \Phi_{\alpha \beta 0} \bar{p}_{\beta}^{b}-\frac{1}{2} \partial_{\alpha} \bar{\Phi}_{\alpha \beta 0} p_{\beta}^{b}+\frac{6}{5} \partial_{\alpha} b_{0} \bar{p}_{\alpha}^{b}+\frac{6}{5} \partial_{\alpha} \bar{b}_{0} p_{\alpha}^{b}-\frac{5}{2} \partial_{\alpha} h_{\alpha 0} \bar{p}^{\varphi}- \\
& -\frac{5}{2} \partial_{\alpha} \bar{h}_{\alpha 0} p^{\varphi}+\frac{7}{48} \bar{\Phi}_{000} p_{\alpha \alpha}^{h}+\frac{7}{48} \Phi_{000} \bar{p}_{\alpha \alpha}^{h}+\bar{\Phi}_{\alpha \beta 0} p_{\alpha \beta}^{h}+\Phi_{\alpha \beta 0} \bar{p}_{\alpha \beta}^{h}- \\
& -\frac{7}{16} \bar{\Phi}_{\alpha \alpha 0} p_{\beta \beta}^{h}-\frac{7}{16} \Phi_{\alpha \alpha 0} \bar{p}_{\beta \beta}^{h}+\frac{3}{40} \bar{b}_{0} p_{\alpha \alpha}^{h}+\frac{3}{40} b_{0} \bar{p}_{\alpha \alpha}^{h}-\frac{5}{4} \bar{\Phi}_{000} p^{\varphi}- \\
& -\frac{5}{4} \Phi_{000} \bar{p}^{\varphi}+\frac{15}{4} \bar{\Phi}_{\alpha \alpha 0} p^{\varphi}+\frac{15}{4} \Phi_{\alpha \alpha 0} \bar{p}^{\varphi}+\frac{9}{2} \bar{b}_{0} p^{\varphi}+\frac{9}{2} b_{0} \bar{p}^{\varphi}+ \\
& +30 \partial_{\beta} \Phi_{\alpha \alpha \beta} \partial_{\delta} \bar{\Phi}_{\gamma \gamma \delta}-30 \partial_{\beta} \Phi_{\alpha \alpha \beta} \partial_{\gamma} \bar{\Phi}_{\gamma 00}-12 \partial_{\beta} \Phi_{\alpha \alpha \beta} \partial_{\gamma} \bar{b}_{\gamma}+ \\
& +10 \partial_{\delta} \Phi_{\alpha \beta \gamma} \partial_{\delta} \bar{\Phi}_{\alpha \beta \gamma}+30 \partial_{\gamma} \Phi_{\alpha \alpha \beta} \partial_{\delta} \bar{\Phi}_{\beta \gamma \delta}-30 \partial_{\gamma} \Phi_{\alpha \alpha \beta} \partial_{\gamma} \bar{\Phi}_{\beta \delta \delta}+ \\
& +30 \partial_{\gamma} \Phi_{\alpha \alpha \beta} \partial_{\gamma} \bar{\Phi}_{\beta 00}+12 \partial_{\gamma} \Phi_{\alpha \alpha \beta} \partial_{\gamma} \bar{b}_{\beta}-30 \partial_{\delta} \bar{\Phi}_{\beta \gamma \delta} \partial_{\alpha} \Phi_{\alpha \beta \gamma}+ \\
& +30 \partial_{\gamma} \bar{\Phi}_{\beta \delta \delta} \partial_{\alpha} \Phi_{\alpha \beta \gamma}-30 \partial_{\gamma} \bar{\Phi}_{\beta 00} \partial_{\alpha} \Phi_{\alpha \beta \gamma}-12 \partial_{\alpha} \Phi_{\alpha \beta \gamma} \partial_{\gamma} \bar{b}_{\beta}- \\
& -12 \partial_{\beta} \bar{\Phi}_{\alpha \alpha \beta} \partial_{\gamma} b_{\gamma}+12 \partial_{\gamma} \bar{\Phi}_{\alpha \alpha \beta} \partial_{\gamma} b_{\beta}-12 \partial_{\alpha} \bar{\Phi}_{\alpha \beta \gamma} \partial_{\gamma} b_{\beta}- \\
& -30 \partial_{\beta} \Phi_{\alpha \alpha 0} \partial_{\gamma} \bar{\Phi}_{\beta \gamma 0}+30 \partial_{\beta} \Phi_{\alpha \alpha 0} \partial_{\beta} \bar{\Phi}_{\gamma \gamma 0}-30 \partial_{\beta} \Phi_{\alpha \alpha 0} \partial_{\beta} \bar{\Phi}_{000}- \\
& -12 \partial_{\beta} \Phi_{\alpha \alpha 0} \partial_{\beta} \bar{b}_{0}+24 \partial_{\gamma} \bar{\Phi}_{\beta \gamma 0} \partial_{\alpha} \Phi_{\alpha \beta 0}-30 \partial_{\beta} \bar{\Phi}_{\gamma \gamma 0} \partial_{\alpha} \Phi_{\alpha \beta 0}+ \\
& +30 \partial_{\beta} \bar{\Phi}_{000} \partial_{\alpha} \Phi_{\alpha \beta 0}+\frac{42}{5} \partial_{\alpha} \Phi_{\alpha \beta 0} \partial_{\beta} \bar{b}_{0}-30 \partial_{\gamma} \Phi_{\alpha \beta 0} \partial_{\gamma} \bar{\Phi}_{\alpha \beta 0}- \\
& -12 \partial_{\beta} \bar{\Phi}_{\alpha \alpha 0} \partial_{\beta} b_{0}+\frac{42}{5} \partial_{\alpha} \bar{\Phi}_{\alpha \beta 0} \partial_{\beta} b_{0}-12 \partial_{\beta} \bar{\Phi}_{\alpha 00} \partial_{\beta} b_{\alpha}- \\
& -30 \partial_{\beta} \Phi_{\alpha 00} \partial_{\gamma} \bar{\Phi}_{\alpha \beta \gamma}+30 \partial_{\beta} \Phi_{\alpha 00} \partial_{\beta} \bar{\Phi}_{\alpha \gamma \gamma}-12 \partial_{\beta} \Phi_{\alpha 00} \partial_{\beta} \bar{b}_{\alpha}- \\
& -30 \partial_{\alpha} \Phi_{\alpha 00} \partial_{\gamma} \bar{\Phi}_{\beta \beta \gamma}+12 \partial_{\alpha} \Phi_{\alpha 00} \partial_{\beta} \bar{b}_{\beta}+12 \partial_{\alpha} \bar{\Phi}_{\alpha 00} \partial_{\beta} b_{\beta}+ \\
& +20 \partial_{\alpha} \bar{\Phi}_{000} \partial_{\alpha} \Phi_{000}+12 \partial_{\alpha} \bar{\Phi}_{000} \partial_{\alpha} b_{0}+30 \partial_{\alpha} \Phi_{000} \partial_{\beta} \bar{\Phi}_{\alpha \beta 0}- \\
& -30 \partial_{\alpha} \Phi_{000} \partial_{\alpha} \bar{\Phi}_{\beta \beta 0}+12 \partial_{\alpha} \Phi_{000} \partial_{\alpha} \bar{b}_{0}-\frac{36}{5} \partial_{\beta} b_{\alpha} \partial_{\alpha} \bar{b}_{\beta}+
\end{aligned}
$$




$$
\begin{aligned}
& +\frac{36}{5} \partial_{\alpha} b_{\alpha} \partial_{\beta} \bar{b}_{\beta}+\frac{216}{25} \partial_{\alpha} b_{0} \partial_{\alpha} \bar{b}_{0}+30 \partial_{\gamma} h_{\alpha \beta} \partial_{\gamma} \bar{h}_{\alpha \beta}-30 \partial_{\gamma} h_{\alpha \beta} \bar{\Phi}_{\alpha \beta \gamma}- \\
& -30 \partial_{\gamma} \bar{h}_{\alpha \beta} \Phi_{\alpha \beta \gamma}+30 \partial_{\beta} h_{\alpha \alpha} \partial_{\gamma} \bar{h}_{\beta \gamma}-30 \partial_{\beta} h_{\alpha \alpha} \partial_{\beta} \bar{h}_{\gamma \gamma}+5 \partial_{\beta} h_{\alpha \alpha} \partial_{\beta} \bar{\varphi}+ \\
& +30 \partial_{\beta} h_{\alpha \alpha} \partial_{\beta} \bar{h}_{00}+15 \partial_{\beta} h_{\alpha \alpha} \bar{\Phi}_{\beta 00}-15 \partial_{\beta} h_{\alpha \alpha} \bar{\Phi}_{\beta \gamma \gamma}-18 \partial_{\beta} h_{\alpha \alpha} \bar{b}_{\beta}- \\
& -60 \partial_{\gamma} \bar{h}_{\beta \gamma} \partial_{\alpha} h_{\alpha \beta}+30 \partial_{\beta} \bar{h}_{\gamma \gamma} \partial_{\alpha} h_{\alpha \beta}-5 \partial_{\beta} \bar{\varphi} \partial_{\alpha} h_{\alpha \beta}-30 \partial_{\beta} \bar{h}_{00} \partial_{\alpha} h_{\alpha \beta}- \\
& -60 \partial_{\alpha} h_{\alpha \beta} \bar{\Phi}_{\beta 00}+60 \partial_{\alpha} h_{\alpha \beta} \bar{\Phi}_{\beta \gamma \gamma}+5 \partial_{\beta} \bar{h}_{\alpha \alpha} \partial_{\beta} \varphi+15 \partial_{\beta} \bar{h}_{\alpha \alpha} \Phi_{\beta 00}- \\
& -15 \partial_{\beta} \bar{h}_{\alpha \alpha} \Phi_{\beta \gamma \gamma}-18 \partial_{\beta} \bar{h}_{\alpha \alpha} b_{\beta}-5 \partial_{\beta} \varphi \partial_{\alpha} \bar{h}_{\alpha \beta}-60 \partial_{\alpha} \bar{h}_{\alpha \beta} \Phi_{\beta 00}+ \\
& +60 \partial_{\alpha} \bar{h}_{\alpha \beta} \Phi_{\beta \gamma \gamma}+\frac{85}{4} \partial_{\alpha} h_{\alpha 0} \partial_{\beta} \bar{h}_{\beta 0}+\frac{325}{8} \partial_{\alpha} h_{\alpha 0} \bar{\Phi}_{000}-\frac{255}{8} \partial_{\alpha} h_{\alpha 0} \bar{\Phi}_{\beta \beta 0}+ \\
& +\frac{63}{4} \partial_{\alpha} h_{\alpha 0} \bar{b}_{0}-60 \partial_{\beta} h_{\alpha 0} \partial_{\beta} \bar{h}_{\alpha 0}+60 \partial_{\beta} h_{\alpha 0} \bar{\Phi}_{\alpha \beta 0}+60 \partial_{\beta} \bar{h}_{\alpha 0} \Phi_{\alpha \beta 0}+ \\
& +\frac{325}{8} \partial_{\alpha} \bar{h}_{\alpha 0} \Phi_{000}-\frac{255}{8} \partial_{\alpha} \bar{h}_{\alpha 0} \Phi_{\beta \beta 0}+\frac{63}{4} \partial_{\alpha} \bar{h}_{\alpha 0} b_{0}-\frac{1}{4} \partial_{\alpha} \varphi \partial_{\alpha} \bar{\varphi}- \\
& -5 \partial_{\alpha} \varphi \partial_{\alpha} \bar{h}_{00}-\frac{5}{2} \partial_{\alpha} \varphi \bar{\Phi}_{\alpha 00}+\frac{5}{2} \partial_{\alpha} \varphi \bar{\Phi}_{\alpha \beta \beta}-5 \partial_{\alpha} \bar{\varphi} \partial_{\alpha} h_{00}-\frac{5}{2} \partial_{\alpha} \bar{\varphi} \Phi_{\alpha 00}+ \\
& +\frac{5}{2} \partial_{\alpha} \bar{\varphi} \Phi_{\alpha \beta \beta}-45 \partial_{\alpha} \bar{h}_{00} \Phi_{\alpha 00}+15 \partial_{\alpha} \bar{h}_{00} \Phi_{\alpha \beta \beta}+18 \partial_{\alpha} \bar{h}_{00} b_{\alpha}- \\
& -30 \partial_{\alpha} h_{00} \partial_{\beta} \bar{h}_{\alpha \beta}+30 \partial_{\alpha} h_{00} \partial_{\alpha} \bar{h}_{\beta \beta}-45 \partial_{\alpha} h_{00} \bar{\Phi}_{\alpha 00}+15 \partial_{\alpha} h_{00} \bar{\Phi}_{\alpha \beta \beta}+ \\
& +18 \partial_{\alpha} h_{00} \bar{b}_{\alpha}+10 \bar{\Phi}_{\alpha \beta \gamma} \Phi_{\alpha \beta \gamma}+30 \bar{\Phi}_{\beta 00} \Phi_{\alpha \alpha \beta}-\frac{255}{16} \bar{\Phi}_{000} \Phi_{\alpha \alpha 0}+ \\
& +\frac{165}{16} \bar{\Phi}_{000} \Phi_{000}+\frac{63}{8} \bar{\Phi}_{000} b_{0}-\frac{255}{16} \bar{\Phi}_{\alpha \alpha 0} \Phi_{000}-\frac{45}{8} \bar{\Phi}_{\alpha \alpha 0} b_{0}-\frac{45}{8} \Phi_{\alpha \alpha 0} \bar{b}_{0}- \\
& -30 \bar{\Phi}_{\beta \gamma \gamma} \Phi_{\alpha \alpha \beta}+\frac{45}{16} \bar{\Phi}_{\beta \beta 0} \Phi_{\alpha \alpha 0}+30 \bar{\Phi}_{\alpha \beta \beta} \Phi_{\alpha 00}+\frac{63}{8} \Phi_{000} \bar{b}_{0}+ \\
& +45 \bar{h}_{00} h_{\alpha \alpha}-45 \bar{h}_{00} h_{00}+45 \bar{h}_{\alpha \alpha} h_{00}-45 \bar{h}_{\beta \beta} h_{\alpha \alpha}+\frac{81}{20} \bar{b}_{0} b_{0} .
\end{aligned}
$$

\section{ПРИЛОЖЕНИЕ Б}

Каноническая плотность гамильтониана в случае лагранжиана (22) имеет вид

$$
\begin{aligned}
\mathcal{H}= & \frac{1}{10} \bar{p}_{\alpha \beta \gamma}^{\Phi} p_{\alpha \beta \gamma}^{\Phi}-\frac{3}{50} \bar{p}_{\beta \gamma \gamma}^{\Phi} p_{\alpha \alpha \beta}^{\Phi}+\frac{1}{6} \bar{p}_{000}^{\Phi} p_{0}^{b}+\frac{1}{6} \bar{p}_{0}^{b} p_{000}^{\Phi}+\frac{1}{20} \bar{p}_{\alpha \alpha \beta}^{\Phi} p_{\beta}^{b}+ \\
& +\frac{1}{20} \bar{p}_{\beta}^{b} p_{\alpha \alpha \beta}^{\Phi}+\frac{1}{30} \bar{p}_{\alpha \beta}^{h} p_{\alpha \beta}^{h}-\frac{1}{60} \bar{p}_{\beta \beta}^{h} p_{\alpha \alpha}^{h}-\frac{5}{36} \bar{p}_{0}^{b} p_{0}^{b}+\frac{1}{6} \bar{p}_{\alpha}^{b} p_{\alpha}^{b}- \\
& -\partial_{\beta} \Phi_{\alpha \alpha \beta} \bar{p}_{000}^{\Phi}-\partial_{\beta} \bar{\Phi}_{\alpha \alpha \beta} p_{000}^{\Phi}-\partial_{\alpha} \Phi_{\alpha 00} \bar{p}_{000}^{\Phi}-\partial_{\alpha} \bar{\Phi}_{\alpha 00} p_{000}^{\Phi}+ \\
& +\frac{3}{5} \partial_{\alpha} \Phi_{\alpha \beta 0} \bar{p}_{\beta \gamma \gamma}^{\Phi}+\frac{3}{5} \partial_{\alpha} \bar{\Phi}_{\alpha \beta 0} p_{\beta \gamma \gamma}^{\Phi}+\frac{1}{2} \partial_{\alpha} h_{\alpha 0} \bar{p}_{\beta \beta}^{h}+\frac{1}{2} \partial_{\alpha} \bar{h}_{\alpha 0} p_{\beta \beta}^{h}+ \\
& +\frac{1}{4} \bar{\Phi}_{000} p_{\alpha \alpha}^{h}+\frac{1}{4} \Phi_{000} \bar{p}_{\alpha \alpha}^{h}+\bar{\Phi}_{\alpha \beta 0} p_{\alpha \beta}^{h}+\Phi_{\alpha \beta 0} \bar{p}_{\alpha \beta}^{h}-\frac{3}{4} \bar{\Phi}_{\alpha \alpha 0} p_{\beta \beta}^{h}- \\
& -\frac{3}{4} \Phi_{\alpha \alpha 0} \bar{p}_{\beta \beta}^{h}-\frac{5}{3} \partial_{\beta} \Phi_{\alpha \alpha \beta} \bar{p}_{0}^{b}-\frac{5}{3} \partial_{\beta} \bar{\Phi}_{\alpha \alpha \beta} p_{0}^{b}-\frac{1}{2} \partial_{\alpha} \Phi_{\alpha \beta 0} \bar{p}_{\beta}^{b}-\frac{1}{2} \partial_{\alpha} \bar{\Phi}_{\alpha \beta 0} p_{\beta}^{b}+ \\
& +\frac{10}{3} \partial_{\alpha} \Phi_{\alpha 00} \bar{p}_{0}^{b}+\frac{10}{3} \partial_{\alpha} \bar{\Phi}_{\alpha 00} p_{0}^{b}+\partial_{\alpha} b_{\alpha} \bar{p}_{0}^{b}+\partial_{\alpha} \bar{b}_{\alpha} p_{0}^{b}-\frac{1}{2} \partial_{\alpha} v_{\alpha} \bar{p}_{0}^{b}- \\
& -\frac{1}{2} \partial_{\alpha} \bar{v}_{\alpha} p_{0}^{b}-\frac{1}{6} \partial_{\alpha} \bar{v}_{0} p_{\alpha}^{b}-\frac{1}{6} \partial_{\alpha} v_{0} \bar{p}_{\alpha}^{b}-2 \partial_{\alpha} \bar{\Phi}_{000} \partial_{\alpha} v_{0}-2 \partial_{\alpha} \Phi_{000} \partial_{\alpha} \bar{v}_{0}+
\end{aligned}
$$




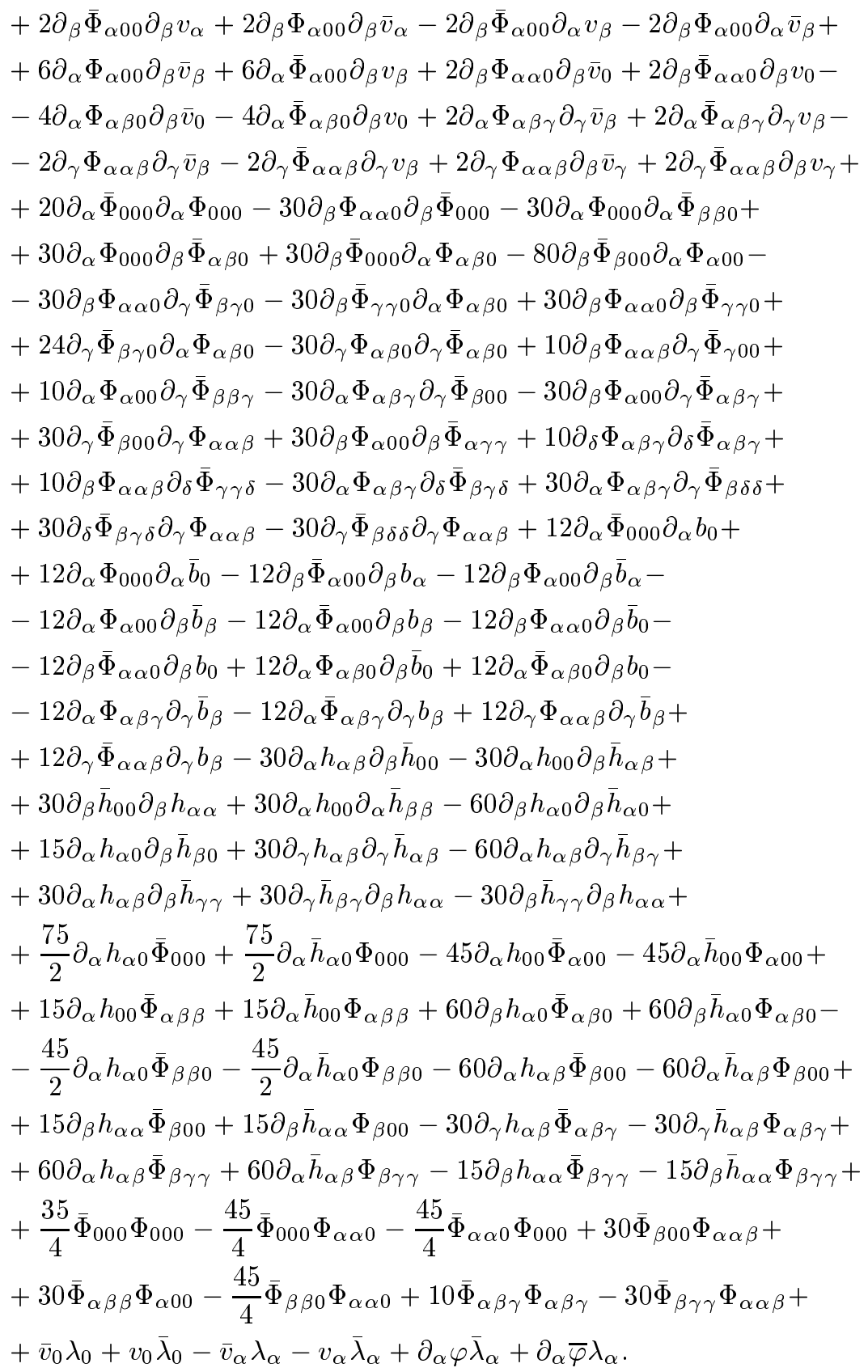

\section{Список литературы}

[1] Yu. M. Zinoviev. Gauge invariant description of massive high spin particles. IHEP preprint 83-91. Protvino: IHEP, 1983.

[2] S. M. Klishevitch, Yu. M. Zinoviev. On electromagnetic interaction of massive spin-2 particle. IHEP preprint 97-41. Protvino: IHEP, 1997 (принята к публикации в ЯФ). 
[3] S. Kamefushi, H. Umesava. Nucl. Phys. 1961. V. 23. P. 399.

[4] S. Kamefushi, L. O. Raifeartaigh, A. Salam. Nucl. Phys. 1961. V. 28. P. 587.

[5] Р. Э. Калои, И. В. Тютин. ЯФ. 1973. Т. 17. С. 190.

[6] A. A. Slavnov. Phys. Lett. B. 1991. V. 258. P. 391.

[7] L. Dolan. Phys. Rev. D. 1984. V. 30. P. 2474.

[8] P. C. Argyres, C. R. Nappi. Phys. Lett. B. 1989. V. 224. P. 89.

[9] D. G. Boulware, S. Deser. Phys. Rev. D. 1972. V. 6. P. 3368.

[10] C. Marzban, B. F. Whiting, H. van Dam. J. Math. Phys. 1989. V. 30. P. 1877.

[11] I. Bengtsson. J. Math. Phys. 1995. V. 36. P. 5805.

[12] P. A. M. Dirac. Proc. Roy. Soc. A. 1958. V. 246. P. 326.

[13] Д. М. Гитман, И. В. Тютин. Каноническое квантование полей со связями. М.: Наука, 1986.

[14] A. A. Slavnov. Nucl. Phys. B. 1971. V. 31. P. 301.

[15] Б. А. Дубровин, С. П. Новиков, А. Т. Фоменко. Современная геометрия: методы и приложения. М.: Наука, 1986.

[16] Y.M. Cho. Spontaneous generation of a massive spin-two field. Preprint SNUTP-92-21. Seul, 1992.

Поступила в редакцию 10.IX.1997 г., после доработки 12.II.1998 г. 